\title{
On Certain Classes of Analytic and Univalent Functions Based on Al-Oboudi Operator
}

\author{
T.V. Sudharsan and S.P. Vijayalakshmi
}

\begin{abstract}
Following the works of [2, 4, 7, 9] of analytic and univalent functions in this paper we introduce two new classes $\mathrm{T}_{\mathrm{n}} \mathrm{S}_{\mathrm{p}}^{\lambda}(\alpha, \beta, \xi, \gamma, \delta)$ and $\mathrm{T}_{\mathrm{n}} \mathrm{V}^{\lambda}(\alpha, \beta, \xi, \gamma, \delta),|z|<1,0<\delta$ $\leq 1, \lambda \geq 0,1 / 2 \leq \xi \leq 1,0 \leq \alpha \leq 1 / 2 \xi, 1 / 2<\gamma \leq 1, \beta \geq 0$ for $a$ family of analytic functions of the form
\end{abstract}

$$
\mathrm{f}(\mathrm{z})=\mathrm{z}-\sum_{\mathrm{n}=2}^{\infty} \mathrm{a}_{\mathrm{n}} \mathrm{z}^{\mathrm{n}}, \mathrm{a}_{\mathrm{n}} \geq 0, \text { for } \mathrm{n}=2,3, \ldots, \mathrm{z} \in \Delta .
$$

We have obtained coefficient estimates, growth \& distortion theorems, extremal properties for these two classes. The determination of extreme points of a family of univalent functions leads to solve many extremal points.

Keywords--- Al-Oboudi Operator, Convex Functions, Starlike Functions, Univalent Functions.

\section{ET S denote the class of functions
$f(z)=z+\sum_{n=2}^{\infty} a_{n} z^{n}$}

\section{INTRODUCTION}

that are analytic and univalent in the disk $|\mathrm{z}|<1$.

For $0 \leq \alpha<1$ let $S^{*}(\alpha)$ and $K(\alpha)$ denote the subfamilies of $S$ consisting of functions starlike of order $\alpha$ and convex of order $\alpha$ respectively.

The subfamily $\mathrm{T}$ of $\mathrm{S}$ consists of functions of the form

$$
\mathrm{f}(\mathrm{z})=\mathrm{z}-\sum_{\mathrm{n}=2}^{\infty} \mathrm{a}_{\mathrm{n}} \mathrm{z}^{\mathrm{n}}, \mathrm{a}_{\mathrm{n}} \geq 0, \text { for } \mathrm{n}=2,3, \ldots, \mathrm{z} \in \Delta .
$$

Silverman [6] investigated function in the classes $\mathrm{T}^{*}(\alpha)=\mathrm{T} \cap \mathrm{S}^{*}(\alpha)$ and $\mathrm{C}(\alpha)=\mathrm{T} \cap \mathrm{K}(\alpha)$.

Let $\mathrm{n} \in \mathrm{N}$ and $\lambda \geq 0$. Denote by $\mathrm{D}_{\lambda}^{\mathrm{n}}$ the Al-Oboudi operator

$$
\begin{aligned}
\quad[3] \text { defined by } D_{\lambda}^{\mathrm{n}}: \mathrm{A} \rightarrow \mathrm{A}, \\
\mathrm{D}_{\lambda}^{0} \mathrm{f}(\mathrm{z})=\mathrm{f}(\mathrm{z}) \\
\mathrm{D}_{\lambda}^{1} \mathrm{f}(\mathrm{z})=(1-\lambda) \mathrm{f}(\mathrm{z})+\lambda z \mathrm{ff}^{\prime}(\mathrm{z})=\mathrm{D}_{\lambda} \mathrm{f}(\mathrm{z}) \\
\mathrm{D}_{\lambda}^{\mathrm{n}} \mathrm{f}(\mathrm{z})=\mathrm{D}_{\lambda}\left[\mathrm{D}_{\lambda}^{\mathrm{n}-1} \mathrm{f}(\mathrm{z})\right] .
\end{aligned}
$$

Note that for $\mathrm{f}(\mathrm{z})$ is given by $(1)$,

$$
D_{\lambda}^{n} f(z)=z+\sum_{j=2}^{\infty}[1+(j-1) \lambda)^{\beta} a_{j} z^{j}, \text { when } \lambda=1,
$$

$\mathrm{D}_{\lambda}^{\mathrm{n}}$ is the Sălăgean differential operator. $\mathrm{D}_{\lambda}^{\mathrm{n}}: \mathrm{A} \rightarrow \mathrm{A}, \mathrm{n} \in$

T.V. Sudharsan, Department of Mathematics, SIVET College, Chennai - 600 073, India. E-mail: tvsudharsan@rediffmail.com

S.P. Vijayalakshmi, Department of Mathematics, SIVET College, Chennai - 600 073, India.
$\mathrm{N}$ defined as:

$$
\begin{aligned}
& D^{0} f(z)=f(z) \\
& D^{1} f(z)=D f(z)=z^{\prime}(z) \\
& D^{n} f(z)=D\left[D^{n-1} f(z)\right]
\end{aligned}
$$

Definition 1.1: [8] Let $\beta, \lambda \in \mathrm{R}, \beta \geq 0, \lambda \geq 0$ and $f(z)=z+\sum_{j=2}^{\infty} a_{j} z^{j}$ we denote by $D_{\lambda}^{\beta}$ the linear operator defined by $\mathrm{D}_{\lambda}^{\beta}: \mathrm{A} \rightarrow \mathrm{A}$

$$
D_{\lambda}^{\beta} f(z)=z+\sum_{j=2}^{\infty}(1+(j-1) \lambda)^{\beta} a_{j} z^{j}
$$

Remark 1.1: If $\mathrm{f} \in \mathrm{T}, \mathrm{f}(\mathrm{z})=\mathrm{z}-\sum_{\mathrm{j}=2}^{\infty} \mathrm{a}_{\mathrm{j}} \mathrm{z}^{\mathrm{j}}, \mathrm{a}_{\mathrm{j}} \geq 0, \mathrm{j}=2,3$, $\ldots$, $\mathrm{z} \in \Delta$ then

$$
D_{\lambda}^{\beta} f(z)=z-\sum_{j=2}^{\infty}(1+(j-1) \lambda)^{\beta} a_{j} z^{j} .
$$

In this paper using the operator $D_{\lambda}^{\beta}$ we introduce the classes $\mathrm{T}_{\mathrm{n}} \mathrm{S}_{\mathrm{p}}^{\lambda}(\alpha, \beta, \xi, \gamma, \delta)$ and $\mathrm{T}_{\mathrm{n}} \mathrm{V}^{\lambda}(\alpha, \beta, \xi, \gamma, \delta)$ and obtain coefficient estimates for these classes when the functions have negative coefficients. We also obtain growth and distortion theorems, closure theorem for functions in these classes.

Definition 1.2: We say that a function $\mathrm{f}(\mathrm{z}) \in \mathrm{T}$ is in the class $\mathrm{T}_{\mathrm{n}} \mathrm{S}_{\mathrm{p}}^{\lambda}(\alpha, \beta, \xi, \gamma, \delta)$ if and only if

$$
\left|\frac{\frac{D_{\lambda}^{\beta+1} \mathrm{f}(\mathrm{z})}{\mathrm{D}_{\lambda}^{\beta} \mathrm{f}(\mathrm{z})}-1}{2 \xi\left(\frac{\mathrm{D}_{\lambda}^{\beta+1} \mathrm{f}(\mathrm{z})}{\mathrm{D}_{\lambda}^{\beta} \mathrm{f}(\mathrm{z})}-\alpha\right)-\gamma\left(\frac{\mathrm{D}_{\lambda}^{\beta+1} \mathrm{f}(\mathrm{z})}{\mathrm{D}_{\lambda}^{\beta} \mathrm{f}(\mathrm{z})}-1\right)}\right|<\delta
$$

where $|z|<1,0<\delta \leq 1,1 / 2 \leq \xi \leq 1, \lambda \geq 0,0 \leq \alpha \leq 1 / 2 \xi$, $1 / 2<\gamma \leq 1, \beta \geq 0$.

Definition 1.3: A function $\mathrm{f}(\mathrm{z}) \in \mathrm{T}$ is said to belong to the class $\mathrm{T}_{\mathrm{n}} \mathrm{S}_{\mathrm{p}}^{\lambda}(\alpha, \beta, \xi, \gamma, \delta)$ if and only if

$$
\left|\frac{\frac{D_{\lambda}^{\beta+2} \mathrm{f}(\mathrm{z})}{\mathrm{D}_{\lambda}^{\beta+1} \mathrm{f}(\mathrm{z})}-1}{2 \xi\left(\frac{\mathrm{D}_{\lambda}^{\beta+2} \mathrm{f}(\mathrm{z})}{\mathrm{D}_{\lambda}^{\beta+1} \mathrm{f}(\mathrm{z})}-\alpha\right)-\gamma\left(\frac{\mathrm{D}_{\lambda}^{\beta+2} \mathrm{f}(\mathrm{z})}{\mathrm{D}_{\lambda}^{\beta+1} \mathrm{f}(\mathrm{z})}-1\right)}\right|<\delta
$$


where $|z|<1,0<\delta \leq 1,1 / 2 \leq \xi \leq 1, \lambda \geq 0,0 \leq \alpha \alpha 1 / 2 \xi$, $1 / 2<\gamma \leq 1, \beta \geq 0$.

If we replace $\beta=0, \lambda=1$ we obtain the corresponding results of S.M. Khairnar and Meena More [4]. If we replace $\beta=0, \lambda=1$ and $\gamma=1$ we obtain the results of Aghalary and Kulkarni [2] and Silverman and Silvia [7]. If we replace $\beta=0, \lambda=1$ and $\xi$ by 1 , we obtain the corresponding results of [9].

\section{MAIN ReSUlts COEFFICIENT Estimates}

Theorem 2.1: A function $\mathrm{f}(\mathrm{z})=\mathrm{z}-\sum_{\mathrm{j}=2}^{\infty} \mathrm{a}_{\mathrm{n}} \mathrm{z}^{\mathrm{n}} \quad\left(\mathrm{a}_{\mathrm{n}} \geq 0\right)$ is in $\mathrm{T}_{\mathrm{n}} \mathrm{S}_{\mathrm{p}}^{\lambda}(\alpha, \beta, \xi, \gamma, \delta)$ if and only if

$$
\begin{gathered}
\sum_{\mathrm{j}=2}^{\infty}(1+(\mathrm{j}-1) \lambda)^{\beta}[(\mathrm{j}-1) \lambda\{2 \xi \delta+1-\gamma \delta\}+2 \delta \xi(1-\alpha)] \mathrm{a}_{\mathrm{j}} \\
\leq 2 \xi \delta(1-\alpha)
\end{gathered}
$$

Proof. Suppose,

$$
\begin{gathered}
\sum_{j=2}^{\infty}(1+(j-1) \lambda)^{\beta}[(j-1) \lambda\{2 \xi \delta+1-\gamma \delta\}+2 \delta \xi(1-\alpha)] a_{j} \\
\leq 2 \xi \delta(1-\alpha)
\end{gathered}
$$

we have

$$
\begin{aligned}
& \left|D_{\lambda}^{\beta+1} f(z)-D_{\lambda}^{\beta} f(z)\right| \\
& -\delta\left|2 \xi\left(D_{\lambda}^{\beta+1} f(z)-\alpha D_{\lambda}^{\beta} f(z)\right)-\gamma\left(D_{\lambda}^{\beta+1} f(z)-D_{\lambda}^{\beta} f(z)\right)\right|<0
\end{aligned}
$$

With the provision,

$$
\begin{aligned}
& \left|z-\sum_{j=2}^{\infty}(1+(j-1) \lambda)^{\beta+1} a_{j} z^{j}-z+\sum_{j=2}^{\infty}(1+(j-1) \lambda)^{\beta} a_{j} z^{j}\right|- \\
& \delta \mid 2 \xi\left[z-\sum_{j=2}^{\infty}(1+(j-1) \lambda)^{\beta+1} a_{j} z^{j}-\alpha z+\alpha \sum_{j=2}^{\infty}(1+(j-1) \lambda)^{\beta} a_{j} z^{j}\right] \\
& -\gamma\left[z-\sum_{j=2}^{\infty}(1+(j-1) \lambda)^{\beta+1} a_{j} z^{j}-z+\sum_{j=2}^{\infty}(1+(j-1) \lambda)^{\beta} a_{j} z^{j}\right] \mid<0
\end{aligned}
$$

For $|\mathrm{z}|=\mathrm{r}<1$ it is bounded above by

$$
\begin{aligned}
& \sum_{\mathrm{j}=2}^{\infty}(1+(\mathrm{j}-1) \lambda)^{\beta}(\mathrm{j}-1) \lambda \mathrm{a}_{\mathrm{j}} \mathrm{r}^{\mathrm{j}}-2 \delta \xi \mathrm{r}(1-\alpha) \\
& -\delta \sum_{\mathrm{j}=2}^{\infty}(1+(\mathrm{j}-1) \lambda)^{\beta}\{2 \xi \alpha-2 \xi-2 \xi(\mathrm{j}-1) \lambda+\gamma(\mathrm{j}-1) \lambda\} \mathrm{a}_{\mathrm{j}} \mathrm{r}^{\mathrm{j}} \\
& \leq 2 \delta \xi(1-\alpha) \\
& \mathrm{f}(\mathrm{z}) \in \mathrm{T}_{\mathrm{n}} \mathrm{S}_{\mathrm{p}}^{\lambda}(\alpha, \beta, \xi, \gamma, \delta) .
\end{aligned}
$$

Now we prove the converse result.

Let

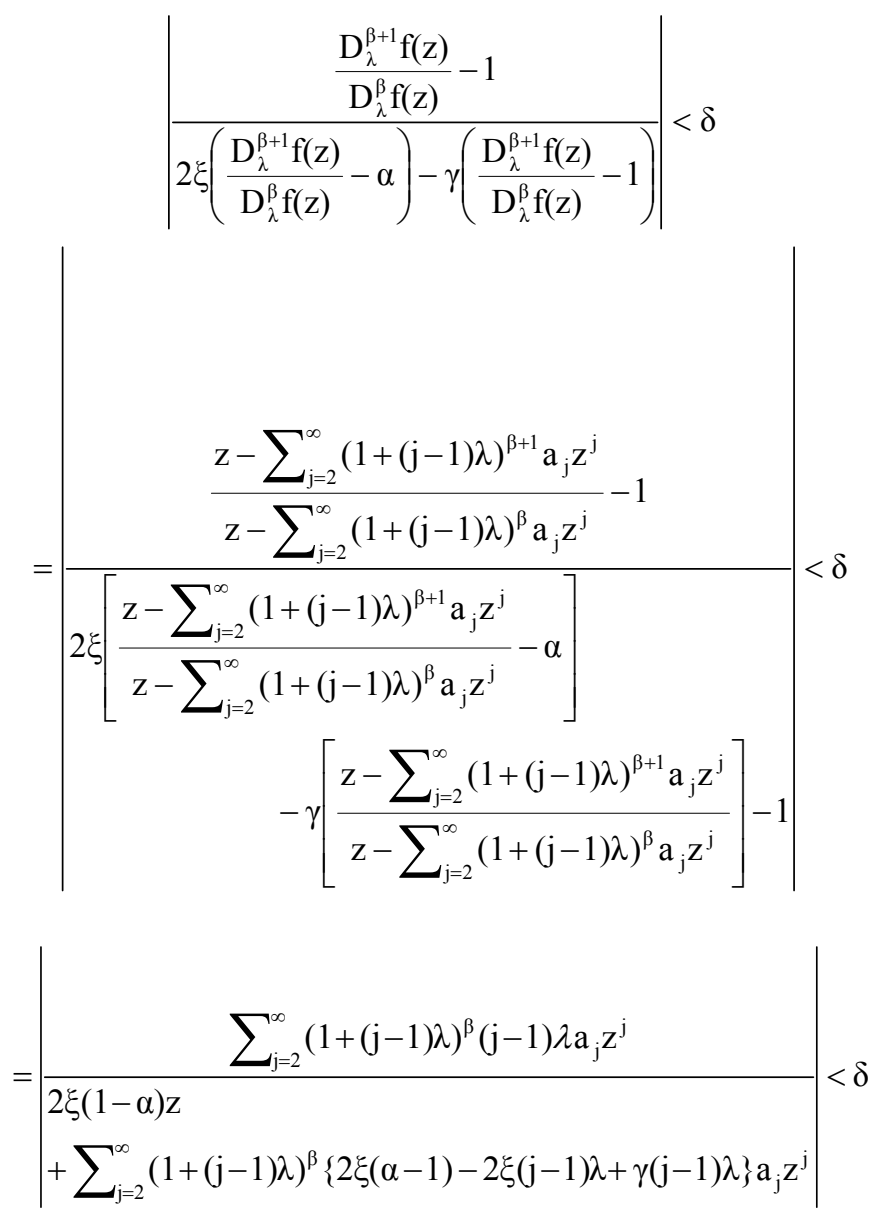

As $|\operatorname{Re} \mathrm{f}(\mathrm{z})| \leq|\mathrm{z}|$ for all $\mathrm{z}$, we have

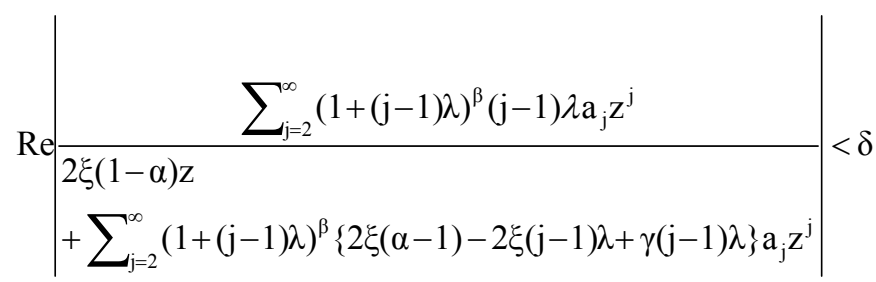

We choose values of $z$ on real axis such that $\frac{D_{\lambda}^{\beta+1}}{D_{\lambda}^{\beta}}$ is real and clearing the denominator of above expression and allowing

$\mathrm{z} \rightarrow 1$ through real values, we obtain

$$
\begin{gathered}
\sum_{\mathrm{j}=2}^{\infty}(1+(\mathrm{j}-1) \lambda)^{\beta}\{(\mathrm{j}-1) \lambda(2 \xi \delta+1-\gamma \delta)+2 \delta \xi(1-\alpha)\} \mathrm{a}_{\mathrm{j}} \\
\leq 2 \xi \delta(1-\alpha) \\
\Rightarrow \sum_{\mathrm{j}=2}^{\infty}\left[(1+(\mathrm{j}-1) \lambda)^{\beta}\{(\mathrm{j}-1) \lambda(2 \xi \delta+1-\gamma \delta)+2 \delta \xi(1-\alpha)\} \mathrm{a}_{\mathrm{j}}\right. \\
2 \xi \delta(1-\alpha)] \leq 0
\end{gathered}
$$

Remark: If $\mathrm{f}(\mathrm{z}) \in \mathrm{T}_{\mathrm{n}} \mathrm{S}_{\mathrm{p}}^{\lambda}(\alpha, \beta, \xi, \gamma, \delta)$ then 


$$
\mathrm{a}_{\mathrm{j}} \leq \frac{2 \xi \delta(1-\alpha)}{(1+(\mathrm{j}-1) \lambda)^{\beta}\{(\mathrm{j}-1) \lambda(2 \xi \delta+1-\gamma \delta)+2 \delta \xi(1-\alpha)\}},
$$

and equality holds for

$f(z)=z-\frac{2 \xi \delta(1-\alpha)}{(1+(j-1) \lambda)^{\beta}\{(j-1) \lambda(2 \xi \delta+1-\gamma \delta)+2 \delta \xi(1-\alpha)\}} z^{j}$.

Corollary 2.1: If $\mathrm{f}(\mathrm{z}) \in \mathrm{T}_{\mathrm{n}} \mathrm{S}_{\mathrm{p}}^{1}(\alpha, 0, \xi, \gamma, \delta)$, that is, replacing $\beta=0, \lambda=1$ we get

$$
\mathrm{a}_{\mathrm{j}} \leq \frac{2 \xi \delta(1-\alpha)}{(\mathrm{j}-1)-\delta(2 \xi \alpha-2 \xi \mathrm{j}+\gamma \mathrm{j}-\gamma)}, \mathrm{j}=2,3,4, \ldots
$$

and equality holds for

$$
f(z)=z-\frac{2 \xi \delta(1-\alpha)}{(j-1)-\delta(2 \xi \alpha-2 \xi j+\gamma j-\gamma)} z^{j} .
$$

This corollary is due to [4].

Corollary 2.2: If $\mathrm{f}(\mathrm{z}) \in \mathrm{T}_{\mathrm{n}} \mathrm{S}_{\mathrm{p}}^{1}(\alpha, 0, \xi, 1, \delta)$, that is, replacing $\beta=0, \lambda=1, \gamma=1$ we get

$$
a_{j} \leq \frac{2 \xi \delta(1-\alpha)}{(j-1)-\delta(2 \xi \alpha-2 \xi j+j-1)}, j=2,3,4, \ldots
$$

and equality holds for

$$
f(z)=z-\frac{2 \xi \delta(1-\alpha)}{(j-1)-\delta(2 \xi \alpha-2 \xi j+j-1)} z^{j} .
$$

This corollary is due to [2] and [7].

Corollary 2.3: If $\mathrm{f}(\mathrm{z}) \in \mathrm{T}_{\mathrm{n}} \mathrm{S}_{\mathrm{p}}^{1}(\alpha, 0,1,1, \delta)$ we get

$$
a_{j} \leq \frac{2 \delta(1-\alpha)}{(j-1)-\delta(2 \alpha-j-1)}, \quad j=2,3,4, \ldots
$$

and equality holds for

$$
f(z)=z-\frac{2 \delta(1-\alpha)}{(j-1)-\delta(2 \alpha-j-1)} z^{j}
$$

This corollary is due to [9].

Corollary 2.4: $\mathrm{f}(\mathrm{z}) \in \mathrm{T}_{\mathrm{n}} \mathrm{S}_{\mathrm{p}}^{1}(\alpha, 0,1,1,1)$ if and only if

$$
\sum_{j=2}^{\infty}(j-\alpha) a_{j} \leq(1-\alpha)
$$

Theorem 2.2: A function $f(z)=z-\sum_{j=2}^{\infty} a_{n} z^{n} \quad\left(a_{n} \geq 0\right)$ is in $\mathrm{T}_{\mathrm{n}} \mathrm{S}_{\mathrm{p}}^{\lambda}(\alpha, \beta, \xi, \gamma, \delta)$ if and only if

$$
\sum_{\mathrm{j}=2}^{\infty}[1+(\mathrm{j}-1) \lambda]^{\beta+1}\{(\mathrm{j}-1) \lambda\{2 \delta \xi+1-\gamma \delta\}+2 \delta \xi(1-\alpha)\} \mathrm{a}_{\mathrm{j}}
$$$$
\leq 2 \delta \xi(1-\alpha) \text {. }
$$

Proof: The proof of this theorem is analogous to that of Theorem [1], because a function $\mathrm{f}(\mathrm{z}) \in \mathrm{T}_{\mathrm{n}} \mathrm{V}^{\lambda}(\alpha, \beta, \xi, \gamma, \delta)$ if and only if $\mathrm{zf}^{\prime}(\mathrm{z}) \in \mathrm{T}_{\mathrm{n}} \mathrm{S}_{\mathrm{p}}^{\lambda}(\alpha, \beta, \xi, \gamma, \delta)$. So it is enough that $\beta$ in Theorem 2.1 is replaced with $\beta+1$.

Remark 2.2: If $\mathrm{f}(\mathrm{z}) \in \mathrm{T}_{\mathrm{n}} \mathrm{V}^{\lambda}(\alpha, \beta, \xi, \gamma, \delta)$ then

$$
\mathrm{a}_{\mathrm{j}} \leq \frac{2 \xi \delta(1-\alpha)}{(1+(\mathrm{j}-1) \lambda)^{\beta+1}\{(\mathrm{j}-1) \lambda(2 \xi \delta+1-\gamma \delta)+2 \delta \xi(1-\alpha)\}},
$$

and equality holds for

$$
f(z)=z-\frac{2 \xi \delta(1-\alpha)}{(1+(j-1) \lambda)^{\beta+1}\{(j-1) \lambda(2 \xi \delta+1-\gamma \delta)+2 \delta \xi(1-\alpha)\}} z^{j}
$$

Corollary 2.5: If $\mathrm{f}(\mathrm{z}) \in \mathrm{T}_{\mathrm{n}} \mathrm{V}^{1}(\alpha, \beta, \xi, \gamma, \delta)$, that is, replacing

$\beta=0, \lambda=1$ we get

$$
\mathrm{a}_{\mathrm{j}} \leq \frac{2 \xi \delta(1-\alpha)}{\mathrm{j}\{(\mathrm{j}-1)-\delta(2 \xi \alpha-2 \xi \mathrm{j}+\gamma \mathrm{j}-\gamma)\}}, \mathrm{j}=2,3,4, \ldots
$$

and equality holds for

$$
f(z)=z-\frac{2 \xi \delta(1-\alpha)}{j\{(j-1)-\delta(2 \xi \alpha-2 \xi j+\gamma j-\gamma)\}} z^{j}
$$

This corollary is due to [4].

Corollary 2.6: If $\mathrm{f}(\mathrm{z}) \in \mathrm{T}_{\mathrm{n}} \mathrm{V}^{1}(\alpha, 0, \xi, 1, \delta)$, that is, replacing

$\beta=0, \lambda=1, \gamma=1$ we get

$$
\mathrm{a}_{\mathrm{j}} \leq \frac{2 \xi \delta(1-\alpha)}{\mathrm{j}\{(\mathrm{j}-1)-\delta(2 \xi \alpha-2 \xi \mathrm{j}+\mathrm{j}-1)\}}, \mathrm{j}=2,3,4, \ldots
$$

and equality holds for

$$
f(z)=z-\frac{2 \xi \delta(1-\alpha)}{j\{(j-1)-\delta(2 \xi \alpha-2 \xi j+j-1)\}} z^{j}
$$

This corollary is due to [2] and [7].

Corollary 2.7: If $\mathrm{f}(\mathrm{z}) \in \mathrm{T}_{\mathrm{n}} \mathrm{V}^{1}(\alpha, 0,1,1, \delta)$, then

$$
a_{j} \leq \frac{2 \delta(1-\alpha)}{j\{(j-1)-\delta(2 \alpha-j-1)\}}, \quad j=2,3,4, \ldots
$$

and equality holds for

$$
f(z)=z-\frac{2 \delta(1-\alpha)}{j\{(j-1)-\delta(2 \alpha-j-1)\}} z^{j}
$$

Corollary 2.8: $\mathrm{f}(\mathrm{z}) \in \mathrm{T}_{\mathrm{n}} \mathrm{V}^{1}(\alpha, 0,1,1,1)$, if and only if

$$
\sum_{j=2}^{\infty} \mathrm{j}(\mathrm{j}-\alpha) \mathrm{a}_{\mathrm{j}} \leq(1-\alpha)
$$


III. GROWTH AND DISTORTION THEOREM

Theorem 3.1: If $\mathrm{f}(\mathrm{z}) \in \mathrm{T}_{\mathrm{n}} \mathrm{S}_{\mathrm{p}}^{\lambda}(\alpha, \beta, \xi, \gamma, \delta)$ then

$$
\begin{array}{r}
\mathrm{r}-\left[\frac{2 \xi \delta(1-\alpha)}{(1+\lambda)^{\beta}\{\lambda+2 \xi \delta \lambda-\gamma \delta \lambda+2 \xi \delta(1-\alpha)\}}\right] \mathrm{r}^{2} \leq|\mathrm{f}(\mathrm{z})| \\
\quad \leq \mathrm{r}+\mathrm{r}^{2}\left[\frac{2 \xi \delta(1-\alpha)}{(1+\lambda)^{\beta}\{\lambda+2 \xi \delta \lambda-\gamma \delta \lambda+2 \xi \delta(1-\alpha)\}}\right]
\end{array}
$$

equality holds for

$$
\mathrm{f}(\mathrm{z})=\mathrm{z}-\frac{2 \xi \delta(1-\alpha)}{1+4 \xi \delta-\delta(\gamma+2 \xi \alpha)} \mathrm{z}^{2} \text { at } \mathrm{z}= \pm \mathrm{r} .
$$

Proof: By Theorem 2.1, we have $\mathrm{f}(\mathrm{z}) \in \mathrm{T}_{\mathrm{n}} \mathrm{S}_{\mathrm{p}}^{\lambda}(\alpha, \beta, \xi, \gamma, \delta)$ if and only if

$$
\begin{gathered}
\sum_{\mathrm{j}=2}^{\infty}(1+(\mathrm{j}-1) \lambda)^{\beta}\{(\mathrm{j}-1) \lambda\{2 \xi \delta+1-\gamma \lambda\}+2 \xi \delta(1-\alpha)\} \mathrm{a}_{\mathrm{j}} \\
\leq 2 \xi \delta(1-\alpha) .
\end{gathered}
$$

Let $\mathrm{t}=1-\frac{2 \xi \delta(1-\alpha)}{\lambda+2 \xi \delta \lambda-\gamma \delta \lambda}$.

$\therefore \mathrm{f}(\mathrm{z}) \in \mathrm{T}_{\mathrm{n}} \mathrm{S}_{\mathrm{p}}^{\lambda}(\alpha, \beta, \xi, \gamma, \delta)$ if and only if

$$
\sum_{j=2}^{\infty}(1+(j-1) \lambda)^{\beta} a_{j}(j-t) \leq 1-t
$$

When $\mathrm{j}=2$

$$
(1+\lambda)^{\beta}(2-t) \sum_{j=2}^{\infty} a_{j} \leq \sum_{j=2}^{\infty}(1+(j-1) \lambda)^{\beta} a_{j}(j-t) \leq 1-t
$$

This last inequality follows from (3) we obtain

$$
|\mathrm{f}(\mathrm{z})| \leq \mathrm{r}+\sum_{\mathrm{j}=2}^{\infty} \mathrm{a}_{\mathrm{n}} \mathrm{r}^{\mathrm{n}} \leq \mathrm{r}+\mathrm{r}^{2} \sum_{\mathrm{j}=2}^{\infty} \mathrm{a}_{\mathrm{n}} \leq \mathrm{r}+\mathrm{r}^{2}\left[\frac{1-\mathrm{t}}{(1+\lambda)^{\beta}(2-\mathrm{t})}\right]
$$

Similarly

$$
|f(z)| \geq r-\sum_{j=2}^{\infty} a_{n} r^{n} \geq r-r^{2} \sum_{j=2}^{\infty} a_{n} \geq r-r^{2}\left[\frac{1-t}{(1+\lambda)^{\beta}(2-t)}\right]
$$

So,

$$
r-r^{2}\left[\frac{1-t}{(1+\lambda)^{\beta}(2-t)}\right] \leq|f(z)| \leq r+r^{2}\left[\frac{1-t}{(1+\lambda)^{\beta}(2-t)}\right]
$$

that is,

$$
\begin{aligned}
\mathrm{r}-\mathrm{r}^{2} & {\left[\frac{2 \xi \delta(1-\alpha)}{(1+\lambda)^{\beta}\{\lambda+2 \xi \delta \lambda-\gamma \delta \lambda+2 \xi \delta(1-\alpha)\}}\right] \leq|\mathrm{f}(\mathrm{z})| } \\
& \leq \mathrm{r}+\mathrm{r}^{2}\left[\frac{2 \xi \delta(1-\alpha)}{(1+\lambda)^{\beta}\{\lambda+2 \xi \delta \lambda-\gamma \delta \lambda+2 \xi \delta(1-\alpha)\}}\right]
\end{aligned}
$$

Hence the result.

Corollary 3.1: If $\mathrm{f} \in \mathrm{T}_{\mathrm{n}} \mathrm{S}_{\mathrm{p}}^{1}(\alpha, 0, \xi, \gamma, \delta)$, that is, replacing $\lambda$ $=1$ and $\beta=0$, then

$$
\begin{array}{r}
\mathrm{r}-\mathrm{r}^{2}\left[\frac{2 \xi \delta(1-\alpha)}{1+2 \xi \delta-\gamma \delta+2 \xi \delta+2 \xi \delta \alpha}\right] \leq|\mathrm{f}(\mathrm{z})| \\
\quad \leq \mathrm{r}+\mathrm{r}^{2}\left[\frac{2 \xi \delta(1-\alpha)}{1+2 \xi \delta-\gamma \delta+2 \xi \delta+2 \xi \delta \alpha}\right]
\end{array}
$$

that is,

$$
\begin{array}{r}
\mathrm{r}-\mathrm{r}^{2}\left[\frac{2 \xi \delta(1-\alpha)}{1+4 \xi \delta-\delta(\gamma+2 \xi \alpha)}\right] \leq|\mathrm{f}(\mathrm{z})| \\
\quad \leq \mathrm{r}+\mathrm{r}^{2}\left[\frac{2 \xi \delta(1-\alpha)}{1+4 \xi \delta-\delta(\gamma+2 \xi \alpha)}\right]
\end{array}
$$

and equality for

$$
\mathrm{f}(\mathrm{z})=\mathrm{z}-\frac{2 \xi \delta(1-\alpha)}{(1+4 \xi \delta)-\delta(\gamma+2 \xi \alpha)} \mathrm{z}^{2} \text { at } \mathrm{z}= \pm \mathrm{r} .
$$

This corollary is due to [4].

Corollary 3.2: If $\mathrm{f}(\mathrm{z}) \in \mathrm{T}_{\mathrm{n}} \mathrm{S}_{\mathrm{p}}^{1}(\alpha, 0, \xi, 1, \delta)$, that is, replacing $\lambda=1, \beta=0$ and $\gamma=1$, then

$$
\begin{array}{r}
\mathrm{r}-\mathrm{r}^{2}\left[\frac{2 \xi \delta(1-\alpha)}{1+4 \xi \delta-\delta(1+2 \xi \alpha)}\right] \leq|\mathrm{f}(\mathrm{z})| \\
\quad \leq \mathrm{r}+\mathrm{r}^{2}\left[\frac{2 \xi \delta(1-\alpha)}{1+4 \xi \delta-\delta(1+2 \xi \alpha)}\right]
\end{array}
$$

with equality for,

$$
f(z)=z-\frac{2 \xi \delta(1-\alpha)}{1+4 \xi \delta-\delta(1+2 \xi \alpha)} z^{j} \quad \text { at } \quad z= \pm r .
$$

This corollary is due to [2] and [7].

Corollary 3.3: If $\mathrm{f}(\mathrm{z}) \in \mathrm{T}_{\mathrm{n}} \mathrm{S}_{\mathrm{p}}^{1}(\alpha, 0,1,1, \delta)$, then

$$
\begin{array}{r}
\mathrm{r}-\mathrm{r}^{2}\left[\frac{2 \delta(1-\alpha)}{1+4 \delta-\delta(1+2 \alpha)}\right] \leq|\mathrm{f}(\mathrm{z})| \\
\quad \leq \mathrm{r}+\mathrm{r}^{2}\left[\frac{2 \delta(1-\alpha)}{1+4 \delta-\delta(1+2 \alpha)}\right]
\end{array}
$$

with equality for,

$$
f(z)=z-\frac{2 \delta(1-\alpha)}{(1+4 \delta)-\delta(1+2 \alpha)} z^{j} \quad \text { at } \quad z= \pm r .
$$

Theorem 3.2: If $\mathrm{f}(\mathrm{z}) \in \mathrm{T}_{\mathrm{n}} \mathrm{V}^{\lambda}(\alpha, \beta, \xi, \gamma, \delta)$ then

$$
\begin{array}{r}
\mathrm{r}-\mathrm{r}^{2}\left[\frac{2 \xi \delta(1-\alpha)}{(1+\lambda)^{\beta+1}\{(\lambda+2 \xi \delta \lambda-\gamma \delta \lambda)+2 \xi \delta(1-\alpha)\}}\right] \leq|\mathrm{f}(\mathrm{z})| \\
\leq \mathrm{r}+\mathrm{r}^{2}\left[\frac{2 \xi \delta(1-\alpha)}{(1+\lambda)^{\beta+1}\{(\lambda+2 \xi \delta \lambda-\gamma \delta \lambda)+2 \xi \delta(1-\alpha)\}}\right]
\end{array}
$$

Proof: The proof of this theorem is analogous to that of Theorem 3.1, because a function $\mathrm{f}(\mathrm{z}) \in \mathrm{T}_{\mathrm{n}} \mathrm{V}^{\lambda}(\alpha, \beta, \chi, \gamma, \delta)$ if and only if $\mathrm{zf}^{\prime}(\mathrm{z}) \in \mathrm{T}_{\mathrm{n}} \mathrm{S}_{\mathrm{p}}^{\lambda}(\alpha, \beta, \xi, \gamma, \delta)$. So it is enough that $\beta$ in Theorem 2.1 is replaced with $\beta+1$. 
Corollary 3.4: If $\mathrm{f}(\mathrm{z}) \in \mathrm{T}_{\mathrm{n}} \mathrm{V}^{1}(\alpha, 0, \xi, \gamma, \delta)$ then

$$
\begin{array}{r}
r-r^{2}\left[\frac{\xi \delta(1-\alpha)}{(1+4 \xi \delta-\delta(\gamma+2 \xi \alpha)}\right] \leq|\mathrm{f}(\mathrm{z})| \\
\quad \leq \mathrm{r}+\mathrm{r}^{2}\left[\frac{\xi \delta(1-\alpha)}{(1+4 \xi \delta-\delta(\gamma+2 \xi \alpha)}\right]
\end{array}
$$

with equality for

$$
f(z)=z-\frac{\xi \delta(1-\alpha)}{(1+4 \xi \delta)-\delta(\gamma+2 \xi \alpha)} z^{j} \text { at } z= \pm r .
$$

This corollary is due to [4].

Corollary 3.5: If $\mathrm{f}(\mathrm{z}) \in \mathrm{T}_{\mathrm{n}} \mathrm{V}^{1}(\alpha, 0, \xi, 1, \delta)$ then

$$
\begin{array}{r}
r-r^{2}\left[\frac{\xi \delta(1-\alpha)}{(1+4 \xi \delta-\delta(1+2 \xi \alpha)}\right] \leq|\mathrm{f}(\mathrm{z})| \\
\quad \leq \mathrm{r}+\mathrm{r}^{2}\left[\frac{\xi \delta(1-\alpha)}{(1+4 \xi \delta-\delta(1+2 \xi \alpha)}\right]
\end{array}
$$

with equality for

$$
f(z)=z-\frac{\xi \delta(1-\alpha)}{(1+4 \xi \delta)-\delta(1+2 \xi \alpha)} z^{j} \text { at } z= \pm r .
$$

This corollary is due to [2] and [7].

Corollary 3.6: If $\mathrm{f}(\mathrm{z}) \in \mathrm{T}_{\mathrm{n}} \mathrm{V}^{1}(\alpha, 0,1,1, \delta)$ then

$$
\begin{array}{r}
r-r^{2}\left[\frac{\delta(1-\alpha)}{1+3 \delta-2 \delta \alpha}\right] \leq|\mathrm{f}(\mathrm{z})| \\
\leq \mathrm{r}+\mathrm{r}^{2}\left[\frac{\delta(1-\alpha)}{1+3 \delta-2 \delta \alpha}\right]
\end{array}
$$

with equality for

$$
f(z)=z-\frac{\delta(1-\alpha)}{1+3 \delta-2 \delta \alpha} z^{j} \text { at } z= \pm r .
$$

This corollary is due to [9].

Theorem 3.3: If $\mathrm{f}(\mathrm{z}) \in \mathrm{T}_{\mathrm{n}} \mathrm{S}_{\mathrm{p}}^{\lambda}(\alpha, \beta, \xi, \gamma, \delta)$ then

$$
\begin{aligned}
1-[ & \left.\frac{4 \xi \delta(1-\alpha)}{(1+\lambda)^{\beta}\{\lambda(1-\gamma \delta)+2 \xi \delta(\lambda+1-\alpha)\}}\right] \mathrm{r} \leq\left|\mathrm{f}^{\prime}(\mathrm{z})\right| \\
& \leq 1+\left[\frac{4 \xi \delta(1-\alpha)}{(1+\lambda)^{\beta}\{\lambda(1-\gamma \delta)+2 \xi \delta(\lambda+1-\alpha)\}}\right] \mathrm{r}
\end{aligned}
$$

Proof. Since $\mathrm{f} \in \mathrm{T}_{\mathrm{n}} \mathrm{S}_{\mathrm{p}}^{\lambda}(\alpha, \beta, \xi, \gamma, \delta)$ we have

$$
\sum_{j=2}^{\infty}(1+(j-1) \lambda)^{\beta} a_{j}(j-t) \leq 1-t
$$

where $\mathrm{t}=1-\frac{2 \xi \delta(1-\alpha)}{\lambda+2 \xi \delta \lambda-\gamma \delta \lambda}$.

In view of Theorem 3.1, we have

$$
\begin{aligned}
& \qquad \sum_{j=2}^{\infty} j a_{j}=\sum_{j=2}^{\infty}(j-t) a_{j}+t \sum_{j=2}^{\infty} a_{j} \\
& \leq \frac{2(1-t)}{(1+\lambda)^{\beta}(2-t)}
\end{aligned}
$$

$$
\left|f^{\prime}\right| \leq 1+\sum_{n=2}^{\infty} n a_{n}|z|^{n-1} \leq 1+r \sum_{n=2}^{\infty} n a_{n} \leq 1+r\left[\frac{2(1-t)}{(1+\lambda)^{\beta}(2-t)}\right]
$$

Similarly

$$
\left|f^{\prime}\right| \geq 1-\sum_{n=2}^{\infty} n a_{n}|z|^{n-1} \geq 1-r \sum_{n=2}^{\infty} n a_{n} \geq 1-r\left[\frac{2(1-t)}{(1+\lambda)^{\beta}(2-t)}\right]
$$

So,

$$
1-r\left[\frac{2(1-t)}{(1+\lambda)^{\beta}(2-t)}\right] \leq\left|f^{\prime}(z)\right| \leq 1+r\left[\frac{2(1-t)}{(1+\lambda)^{\beta}(2-t)}\right] .
$$

Substituting t, we have

$$
\begin{gathered}
1-\left[\frac{4 \xi \delta(1-\alpha)}{(1+\lambda)^{\beta}\{\lambda(1-\gamma \delta)+2 \xi \delta(\lambda+1-\alpha)\}}\right] \mathrm{r} \leq\left|\mathrm{f}^{\prime}(\mathrm{z})\right| \\
\quad \leq 1+\left[\frac{4 \xi \delta(1-\alpha)}{(1+\lambda)^{\beta}\{\lambda(1-\gamma \delta)+2 \xi \delta(\lambda+1-\alpha)\}}\right] \mathrm{r}
\end{gathered}
$$

Corollary 3.7: If $\left.f \in \mathrm{T}_{\mathrm{n}} \mathrm{S}_{\mathrm{p}}^{1}(\alpha, 0, \xi, \gamma, \delta)\right)$ then

$$
\begin{gathered}
1-\left[\frac{4 \xi \delta(1-\alpha)}{(1-\gamma \delta)+2 \xi \delta(2-\alpha)}\right] \mathrm{r} \leq\left|\mathrm{f}^{\prime}(\mathrm{z})\right| \\
\quad \leq 1+\left[\frac{4 \xi \delta(1-\alpha)}{(1-\gamma \delta)+2 \xi \delta(2-\alpha)}\right] \mathrm{r}
\end{gathered}
$$

for $|z|=r$. This corollary is due to [4].

Corollary 3.8: If $\mathrm{f} \in \mathrm{T}_{\mathrm{n}} \mathrm{S}_{\mathrm{p}}^{1}(\alpha, 0, \xi, 1, \delta)$ then

$$
\begin{array}{r}
1-\left[\frac{4 \xi \delta(1-\alpha)}{(1-\delta)+2 \xi \delta(2-\alpha)}\right] \mathrm{r} \leq\left|\mathrm{f}^{\prime}(\mathrm{z})\right| \\
\quad \leq 1+\left[\frac{4 \xi \delta(1-\alpha)}{(1-\delta)+2 \xi \delta(2-\alpha)}\right] \mathrm{r}
\end{array}
$$

This corollary is due to [2] and [7].

Corollary 3.9: If $f \in \mathrm{T}_{\mathrm{n}} \mathrm{S}_{\mathrm{p}}^{1}(\alpha, 0,1,1, \delta)$ then

$$
\begin{gathered}
1-\left[\frac{4 \delta(1-\alpha)}{(1-\delta)+2 \delta(2-\alpha)}\right] \mathrm{r} \leq\left|\mathrm{f}^{\prime}(\mathrm{z})\right| \\
\leq 1+\left[\frac{4 \delta(1-\alpha)}{(1-\delta)+2 \delta(2-\alpha)}\right] \mathrm{r}
\end{gathered}
$$

that is,

$$
\begin{gathered}
1-\left[\frac{4 \delta(1-\alpha)}{1+3 \delta-2 \delta \alpha}\right] \mathrm{r} \leq\left|\mathrm{f}^{\prime}(\mathrm{z})\right| \\
\leq 1+\left[\frac{4 \delta(1-\alpha)}{1+3 \delta-2 \delta \alpha}\right] \mathrm{r}
\end{gathered}
$$

This corollary is due to [9].

Theorem 3.4: If $\mathrm{f} \in \mathrm{T}_{\mathrm{n}} \mathrm{V}^{\lambda}(\alpha, \beta, \xi, \gamma, \delta)$ then 


$$
\begin{array}{r}
1-\left[\frac{4 \xi \delta(1-\alpha)}{(1+\lambda)^{\beta+1}\{\lambda(1-\gamma \delta)+2 \xi \delta(\lambda+1-\alpha)\}}\right] \mathrm{r} \leq\left|\mathrm{f}^{\prime}(\mathrm{z})\right| \\
\quad \leq 1+\mathrm{r}\left[\frac{4 \xi \delta(1-\alpha)}{(1+\lambda)^{\beta+1}\{\lambda(1-\gamma \delta)+2 \xi \delta(\lambda+1-\alpha)\}}\right]
\end{array}
$$

for $|z|=r$.

Proof: The proof of this theorem is similar to that of Theorem 3.3 because a function $\mathrm{f}(\mathrm{z}) \in \mathrm{T}_{\mathrm{n}} \mathrm{V}^{\lambda}(\alpha, \beta, \xi, \gamma, \delta)$ if and only if $\mathrm{zf}^{\prime}(\mathrm{z}) \in \mathrm{T}_{\mathrm{n}} \mathrm{S}_{\mathrm{p}}^{\lambda}(\alpha, \beta, \xi, \gamma, \delta)$. So it is enough that $\beta$ in Theorem 3.3 is replaced with $\beta+1$.

Corollary 3.10: If $\mathrm{f} \in \mathrm{T}_{\mathrm{n}} \mathrm{V}^{1}(\alpha, 0, \xi, \gamma, \delta)$ then

$$
\begin{array}{r}
1-\left[\frac{2 \xi \delta(1-\alpha)}{(1-\gamma \delta)+2 \xi \delta(2-\alpha)}\right] \mathrm{r} \leq\left|\mathrm{f}^{\prime}(\mathrm{z})\right| \\
\quad \leq 1+\mathrm{r}\left[\frac{2 \xi \delta(1-\alpha)}{(1-\gamma \delta)+2 \xi \delta(2-\alpha)}\right]
\end{array}
$$

for $|z|=r$. This corollary is due to [4].

Corollary 3.11: If $\mathrm{f} \in \mathrm{T}_{\mathrm{n}} \mathrm{V}^{1}(\alpha, 0, \xi, 1, \delta)$ then

$$
\begin{array}{r}
1-\left[\frac{2 \xi \delta(1-\alpha)}{(1-\delta)+2 \xi \delta(2-\alpha)}\right] \mathrm{r} \leq\left|\mathrm{f}^{\prime}(\mathrm{z})\right| \\
\quad \leq 1+\mathrm{r}\left[\frac{2 \xi \delta(1-\alpha)}{(1-\delta)+2 \xi \delta(2-\alpha)}\right]
\end{array}
$$

for $|z|=r$. This corollary is due to [2] and [7].

Corollary 3.12: If $\mathrm{f} \in \mathrm{T}_{\mathrm{n}} \mathrm{V}^{1}(\alpha, 0,1,1, \delta)$ then

$$
\begin{gathered}
1-\left[\frac{2 \delta(1-\alpha)}{(1-\delta)+2 \delta(2-\alpha)}\right] \mathrm{r} \leq\left|\mathrm{f}^{\prime}(\mathrm{z})\right| \\
\leq 1+\mathrm{r}\left[\frac{2 \delta(1-\alpha)}{(1-\delta)+2 \delta(2-\alpha)}\right] \\
1-\left[\frac{2 \delta(1-\alpha)}{1+3 \delta-2 \alpha \delta}\right] \mathrm{r} \leq\left|\mathrm{f}^{\prime}(\mathrm{z})\right| \\
\leq 1+\mathrm{r}\left[\frac{2 \delta(1-\alpha)}{1+3 \delta-2 \alpha \delta}\right]
\end{gathered}
$$

for $|z|=r$. This corollary is due to [9].

\section{Closure Theorem}

Theorem 4.1: Let $\mathrm{f}_{1}(\mathrm{z})=\mathrm{z}$ and

$$
\mathrm{f}_{\mathrm{j}}(\mathrm{z})=\frac{2 \xi \delta(1-\alpha)}{[1+(\mathrm{j}-1) \lambda]^{\beta}[(\mathrm{j}-1) \lambda\{2 \xi \delta+1-\gamma \delta\}+2 \delta \xi(1-\alpha)]} \mathrm{z}^{\mathrm{j}}
$$

for $\mathrm{j}=2,3,4, \ldots$.
Then $\mathrm{f}(\mathrm{z}) \in \mathrm{T}_{\mathrm{n}} \mathrm{V}^{\lambda}(\alpha, \beta, \xi, \gamma, \delta)$ if and only if $\mathrm{f}(\mathrm{z})$ can be expressed in the forms $f(z)=\sum_{j=1}^{\infty} \lambda_{j} f_{j}(z)$ where $\lambda_{j} \geq 0$ and $\sum_{j=1}^{\infty} \lambda_{j}=1$

Proof: Let $f(z)=\sum_{j=1}^{\infty} \lambda_{j} f_{j}(z), \lambda_{j} \geq 0, j=1,2, \ldots$ with $\sum_{j=1}^{\infty} \lambda_{j}=1$ we have

$$
\begin{aligned}
& f(z)=\sum_{j=1}^{\infty} \lambda_{j} f_{j}(z) \\
& =\lambda_{1} f_{1}(z)+\sum_{j=2}^{\infty} \lambda_{j} f_{j}(z) \\
& =z-\sum_{j=2}^{\infty} \lambda_{j}\left[\frac{2 \xi \delta(1-\alpha)}{[1+(j-1) \lambda]^{\beta}[(j-1) \lambda\{2 \xi \delta+1-\gamma \delta\}+2 \delta \xi(1-\alpha)]} z^{j}\right]
\end{aligned}
$$

Then

$$
\begin{aligned}
& \sum_{j=2}^{\infty} \lambda_{j} \frac{2 \xi \delta(1-\alpha)}{[1+(j-1) \lambda]^{\beta}[(j-1) \lambda\{2 \xi \delta+1-\gamma \delta\}+2 \delta \xi(1-\alpha)]} \\
& \quad \times \frac{[1+(j-1) \lambda]^{\beta}[(j-1) \lambda\{2 \xi \delta+1-\gamma \delta\}+2 \delta \xi(1-\alpha)]}{2 \xi \delta(1-\alpha)} \\
& =\sum_{j=2}^{\infty} \lambda_{j}=1-\lambda_{1} \leq 1
\end{aligned}
$$

$\mathrm{f}(\mathrm{z}) \in \mathrm{T}_{\mathrm{n}} \mathrm{V}^{\lambda}(\alpha, \beta, \xi, \gamma, \delta)$.

Conversely, suppose $\mathrm{f}(\mathrm{z}) \in \mathrm{T}_{\mathrm{n}} \mathrm{V}^{\lambda}(\alpha, \beta, \xi, \gamma, \delta)$ then remark of Theorem 2.1 gives us

$$
\mathrm{a}_{\mathrm{j}} \leq \frac{2 \xi \delta(1-\alpha)}{[1+(\mathrm{j}-1) \lambda]^{\beta}[(j-1) \lambda\{2 \xi \delta+1-\gamma \delta\}+2 \delta \xi(1-\alpha)]}
$$

we take

$$
\begin{gathered}
\lambda_{\mathrm{j}}=\frac{[1+(\mathrm{j}-1) \lambda]^{\beta}[(\mathrm{j}-1) \lambda\{2 \xi \delta+1-\gamma \delta\}+2 \delta \xi(1-\alpha)]}{2 \xi \delta(1-\alpha)} \mathrm{a}_{\mathrm{j}}, \\
\mathrm{j}=2,3,4, \ldots
\end{gathered}
$$

and $\lambda_{1}=1-\sum_{\mathrm{j}=1}^{\infty} \lambda_{\mathrm{j}}$.

Then $f(z)=\sum_{j=1}^{\infty} \lambda_{j} f_{j}(z)$.

Corollary 4.1: If $\mathrm{f}_{1}(\mathrm{z})=\mathrm{z}$ and

$$
\mathrm{f}_{\mathrm{j}}(\mathrm{z})=\mathrm{z}-\frac{2 \xi \delta(1-\alpha)}{(\mathrm{j}-1)-\delta(2 \xi \alpha-2 \xi \mathrm{j}+\gamma \mathrm{j}-\gamma)} \mathrm{z}^{\mathrm{j}} \text {, for } \mathrm{j}=2,3, \ldots
$$

Then $\mathrm{f}(\mathrm{z}) \in \mathrm{T}_{\mathrm{n}} \mathrm{V}^{1}(\alpha, 0, \xi, \gamma, \delta)$ if and only if $\mathrm{f}(\mathrm{z})$ can be expressed in the form $f(z)=\sum_{j=1}^{\infty} \lambda_{j} f_{j}(z)$ where $\lambda_{j} \geq 0$, $\mathrm{j}=1,2, \ldots, \sum_{\mathrm{j}=1}^{\infty} \lambda_{\mathrm{j}}=1$.

This corollary is due to [4]. 
Corollary 4.2: If $\mathrm{f}_{1}(\mathrm{z})=\mathrm{z}$ and

$$
f_{j}(z)=z-\frac{2 \xi \delta(1-\alpha)}{(j-1)-\delta(2 \xi \alpha-2 \xi j+j-1)} z^{j}, \text { for } j=2,3, \ldots
$$

Then $\mathrm{f}(\mathrm{z}) \in \mathrm{T}_{\mathrm{n}} \mathrm{V}^{1}(\alpha, 0, \xi, 1, \delta)$ if and only if $\mathrm{f}(\mathrm{z})$ can be expressed in the form $f(z)=\sum_{j=1}^{\infty} \lambda_{j} f_{j}(z)$ where $\lambda_{j} \geq 0$, $\mathrm{j}=1,2, \ldots, \sum_{\mathrm{j}=1}^{\infty} \lambda_{\mathrm{j}}=1$.

This corollary is due to [2] and [7].

Corollary 4.3: If $\mathrm{f}_{1}(\mathrm{z})=\mathrm{z}$ and

$$
f_{j}(z)=z-\frac{2 \delta(1-\alpha)}{(j-1)-\delta(2 \alpha-j-1)} z^{j}, \text { for } j=2,3, \ldots
$$

Then $\mathrm{f}(\mathrm{z}) \in \mathrm{T}_{\mathrm{n}} \mathrm{V}^{1}(\alpha, 0,1,1, \delta)$ if and only if $\mathrm{f}(\mathrm{z})$ can be expressed in the form $f(z)=\sum_{j=1}^{\infty} \lambda_{j} f_{j}(z)$ where $\lambda_{j} \geq 0$, $\mathrm{j}=1,2, \ldots, \sum_{\mathrm{j}=1}^{\infty} \lambda_{\mathrm{j}}=1$.

This corollary is due to [9].

Corollary 4.4: If $\mathrm{f}_{1}(\mathrm{z})=\mathrm{z}$ and

$$
f_{j}(z)=z-\frac{2}{2 j} z^{j}=z-\frac{z^{j}}{j}
$$

Then $f(z) \in T_{n} V^{1}(0,0,1,1,1)$ if and only if $f(z)$ can be expressed in the form $f(z)=\sum_{j=1}^{\infty} \lambda_{j} f_{j}(z)$ where $\lambda_{j} \geq 0$, $j=1,2, \ldots, \sum_{j=1}^{\infty} \lambda_{j}=1$

\section{CONCLUSION}

In this paper making use of Al-oboudi operator two new subclasses of analytic and univalent functions are introduced for the functions with negative coefficients. Many subclasses which are already studied by various researchers are obtained as special cases of our two new subclasses. We have obtained various properties such as coefficient estimates, growth distortion theorems. Further new subclasses may be possible from the two classes introduced in this paper.

\section{REFERENCES}

[1] M. Acu, S. Owa, "Note on a class of starlike functions", Proceeding of the International short work on study on calculus operators in univalent function theory, Kyoto, Pp. 1-10, 2006.

[2] R. Aghalary and S. Kulkarni, "Some theorems on univalent functions", J. Indian Acad. Math., Vol. 24, No. 1, Pp. 81-93, 2002.

[3] F.M. Al-Oboudi, "On univalent functions defined by a generalized Sălăgean operator”, Ind. J. Math. Sci., No. 25-28, 1429-1436, 2004.

[4] S.M. Khairnar and Meena More, "Certain family of analytic and univalent functions", Acta Mathematica Academiae Paedogical, Vol. 24, Pp. 333-344, 2008.

[5] S.R. Kulkarni, "Some problems connected with univalent functions", Ph.D. Thesis, Shiraji University, Kolhapur, 1981.
[6] H. Silverman, "Univalent functions with negative coefficients".

[7] H. Silverman and E. Silvia, "Subclasses of prestarlike functions", Math. Japon, Vol. 29, No. 6, Pp. 929-935, 1984.

[8] T.V. Sudharsan, R. Thirumalaisamy, K.G. Subramanian, Mugur Acu, “A class of analytic functions based on an extension of Al-Oboudi operator", Acta Universitatis Apulensis, Vol. 21, Pp. 79-88, 2010.

[9] S. Owa and J. Nishiwaki, "Coefficient Estimates for certain classes of analytic functions", JIPAM, J. Inequal. Pure Appl. Math., Vol. 315, Article 72, 5pp (electonic), 2002. 